\title{
Sample Thickness Determination By Scanning Transmission Electron Microscopy At Low Electron Energies
}

\author{
T. Volkenandt, E. Müller, D. Gerthsen \\ Laboratory for Electron Microscopy, Karlsruhe Institute of Technology (KIT), Engesserstr. 7, 76131 \\ Karlsruhe, Germany
}

Knowledge of the local sample thickness is essential in TEM for the quantification of almost any image information. Established techniques for the determination of the sample thickness comprise the exploitation of plasmon losses in EELS spectra, convergent beam electron diffraction, electron holography, thickness contours under well-defined excitation conditions and STEM at TEM-typical energies. More recently, high-angle annular dark-field (HAADF) STEM at electron energies $E_{0} \leq 30$ $\mathrm{keV}$ emerged as a promising technique for TEM sample-thickness determination [1-3]. This method is based on the comparison of experimental and calculated HAADF STEM intensities and can be applied for samples with known composition. It is advantageous that low-energy STEM images can be recorded in an easy-to-operate scanning electron microscope fitted with a STEM detector. Combining low-energy STEM in a scanning electron microscope with a focused ion-beam (FIB) system for TEM sample preparation facilitates thickness determination after FIB sample preparation. Moreover, knock-on damage is negligible at low $\mathrm{E}_{0}$ in a scanning electron microscope (SEM).

In this work we show sample thickness determination by low-energy HAADF STEM at electron energies between $15 \mathrm{keV}$ and $30 \mathrm{keV}$ on different sample materials comprising fluorenyl hexa-perihexabenzocoronene (atomic number $Z=3.5$ ), carbon $(Z=6)$, silicon $(Z=14)$, gallium nitride $(Z=19)$ and tungsten $(Z=74)$. Measurements were performed either on films with well-known thickness or wedge-shaped samples obtained by FIB milling. Measured HAADF STEM intensities were normalized with respect to the incident electron intensity and corrected for detector-related effects [3]. The experimental data was compared with Monte-Carlo (MC) simulations performed with the NIST Monte program using screened Rutherford (SR-CSs) or Mott cross-sections (M-CSs) [4].

Fig. 1a shows a cross-section HAADF STEM image of a wedge-shaped sample with an amorphous Clayer on a mica substrate and a Pt/C-protection layer on top. The SEM top-view image Fig. 1b confirms the wedge shape of the sample. The sample thickness $t$ in Fig. 1a increases from left to right due to the wedge geometry of the sample. Figs. 2a,b contain measured HAADF STEM intensity profiles (black solid lines) as a function of the thickness, taken along the white arrow in Fig. 1a, and simulated data. The HAADF STEM intensity $I_{H A A D F}$ is characterized by a maximum for $E_{0}=15 \mathrm{keV}$ (Fig. 2a) which shifts towards smaller thickness for lower $E_{0}$ and materials with higher Z-values. The experimental $\mathrm{I}_{\mathrm{HAADF}}$ can be well described by MC-simulations based on SR-CSs at $30 \mathrm{keV}$ (Fig. 2b). At $15 \mathrm{keV}$, a discrepancy exists between experimental data and $\mathrm{MC}$-simulations irrespective of the used scattering cross-section.

Investigations of the other sample materials reveal that MC-simulations based on M-CSs well describe the experimental data at $30 \mathrm{keV}$ for materials with intermediate and high $\mathrm{Z}$-values as verified for $\mathrm{Z}=14$ (silicon), $19(\mathrm{GaN})$ and 74 (tungsten). The choice of the scattering cross-section is more complex for low-density materials. FHBC $(\mathrm{Z}=3.5)$ at $15 \mathrm{keV}$ and $30 \mathrm{keV}$ as well as carbon at $30 \mathrm{keV}$ can be adequately modelled by MC-simulations based on SR-CSs. Discrepancies between measured and 
simulated $I_{\text {HAADF }}$ are found at $15 \mathrm{keV}$ for materials with Z-values of 6 (carbon) and $19(\mathrm{GaN})$ independent on the chosen model for the scattering cross-section. The source of this discrepancy needs further investigations.

The accuracy of the thickness determination increases with the slope of the $I_{\text {HAADF }}$ vs. $t$ curve. Accordingly, conditions should be avoided where measurements are performed close to the intensity maximum of the $I_{H A A D F}$ vs. $t$ curve where the slope is small. A guideline for the selection of suitable $E_{0^{-}}$ values can be derived by calculating the thickness or mass-thickness, respectively, at maximum $I_{H A A D F}$ as a function of $\mathrm{Z}$ and $E_{0}$. The main error can be the simulated intensities if unfavorable conditions are chosen (scattering cross-sections, $\mathrm{E}_{0}$-values). This error can be excluded if $I_{\text {HAADF }} \mathrm{vs}$. $t$ calibration curves are acquired using TEM samples with a known thickness profile like the wedge samples in this work. Ambiguities due to the maximum of the $I_{\text {HAADF }}$ vs. $t$ curves can be avoided if measurements are performed at two different $\mathrm{E}_{0}$-values. Choosing proper conditions, the overall accuracy of the method is better than $\pm 5 \%$ if the sample thickness is homogeneous in the analyzed region [5].

\section{References:}

[1] V Morandi, P Merli, J. Appl. Phys. 101 (2007) p. 114917.

[2] V Krzyzanek, R Reichelt, Conference Proceedings of the 14th European Microscopy Congress (2008), 1-5 September 2008, Aachen.

[3] T Volkenandt, E Müller, D Gerthsen, Microsc. Microanal., in press.

[4] N Ritchie, Surf. Interf. Anal. 37 (2005), p. 1006.

[5] Acknowledgement: This work has been supported by the Deutsche Forschungsgemeinschaft (DFG).
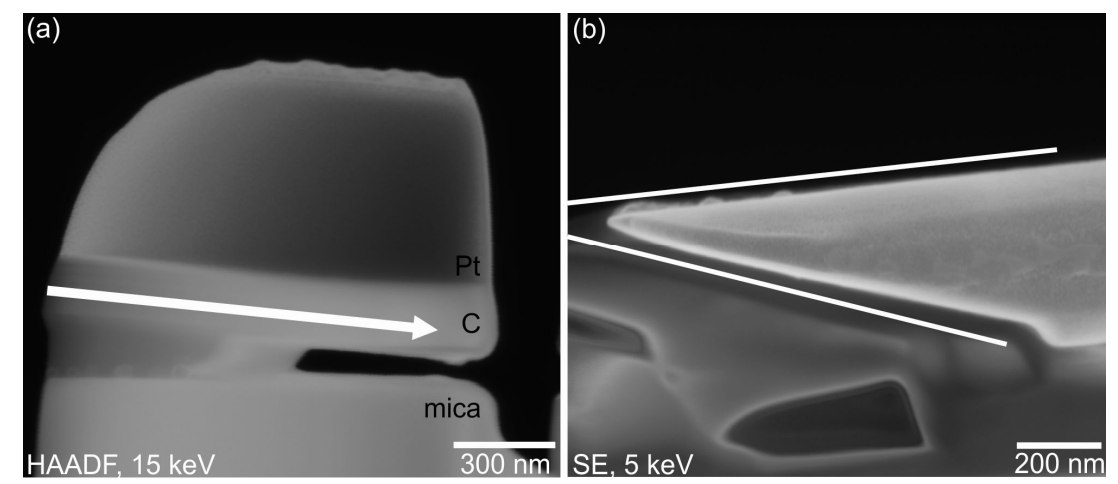

Figure 1. (a) $15 \mathrm{keV}$ cross-section HAADF STEM image of an amorphous carbon layer (white arrow) with a wedge-shaped thickness profile, (b) $5 \mathrm{keV} \mathrm{SEM}$ top-view image of the wedge sample with indicated wedge angle.
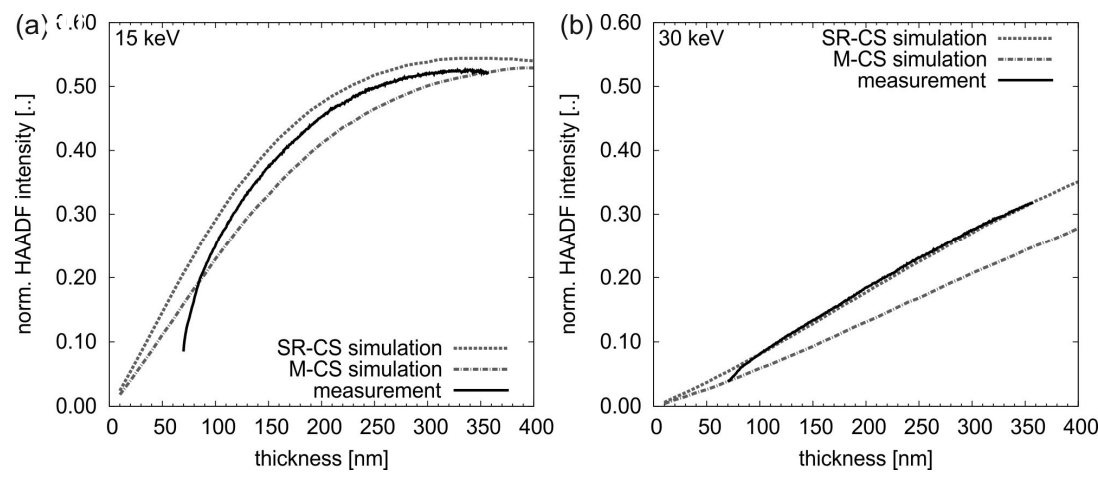

Figure 2. Comparison of measure (solid black lines) and simulated HAADF STEM intensities of amorphous carbon as a function of the sample thickness for (a) $15 \mathrm{keV}$ and (b) $30 \mathrm{keV}$. MC-simulations are performed with screened Rutherford (dotted lines) and Mott cross-sections (dashed lines). 